\title{
Identification and Characterization of 12 Mitogen- activated Protein Kinase Genes Implicated in Stress Responses in Cherry Rootstocks
}

\author{
Xiaojuan Zong1, Jiawei Wang, Li Xu, Hairong Wei, Xin Chen, Dongzi Zhu, Yue Tan, \\ and Qingzhong Liu ${ }^{1}$ \\ Key Laboratory for Fruit Biotechnology Breeding of Shandong Province, Shandong Institute of \\ Pomology, Shandong Academy of Agricultural Sciences, Taian, Shandong Province, 271000, China
}

\begin{abstract}
AdDitional INDEX wORDs. 'Gisela 6', gene identification, expression profiling, abiotic and biotic stresses
Abstract. Improving the poor resistance to environmental stress and the weak development of roots system in the cherry (Prunus) rootstock 'Gisela 6' (Prunus cerasus $\times$ Prunus canescens) is of great importance for sustainable sweet cherry (Prunus avium) production. Although a stable genetic transformation system has been developed for 'Gisela 6' rootstock, there is little information on the identification of genes involved in stress resistance. Using the cherry rootstock cultivar Gisela 6, we identified a total of 12 novel mitogen-activated protein kinase (MAPK) genes, designated PcMPKs. Phylogenetic analysis revealed that the PcMPKs could be divided into four groups, designated A, $B, C$, and D. In addition, an intron-exon structure analysis for the PcMPKs was conducted to help further understand the structure-function relationships within the cherry family. The expression profiles of $P c M P K s$ in response to abiotic and biotic stresses were characterized using quantitative reverse transcription-polymerase chain reaction (qRT-PCR). Five PcMPKs (i.e., PcMPK4-1, PcMPK4-2, PcMPK3, PcMPK6, and PcMPK18) exhibited differential expression, and suggested their potential roles in plant responding to various stresses. This study provides the basis for further analysis on the physiological functions of PcMPKs in environmental tolerance in cherry rootstocks.
\end{abstract}

\begin{abstract}
'Gisela' rootstock, a triploid hybrid of $P$. cerasus and $P$. canescens, is currently the most popular dwarf rootstock for sweet cherry (Koc et al., 2013; Usenik et al., 2010). However, the main disadvantages in this cultivar are the weak development of root systems and poor resistance to environmental stresses such as cold, drought, salinity, or pathogen infection. Conventional approaches for cherry rootstock improvement are generally difficult and occur over long time frames as a result of the heterozygosity and polyploidy of the cross. Genetic transformation with a single gene or multiple genes offers an attractive approach to efficiently improve the problems existing in this cultivar. Although a stable genetic transformation system has been developed for 'Gisela 6' (Song, 2014; Song and Sink, 2006; Song et al., 2013; Zhao and Song, 2014), little has been reported on gene identification for this cultivar.

MAPK cascades are highly conserved signal transduction modules that play crucial roles in regulating many biological processes in plants, such as growth, development, and responses to biotic and abiotic stresses (Ligterink, 2000; Ligterink and Hirt, 2001; MAPK Group, 2002; Mizoguchi et al., 2000; Nakagami et al., 2005; Zhang et al., 2006; Zwerger and Hirt, 2001). MAPKs are dual-specificity protein kinases that are activated by mitogen-activated protein kinase kinases through double phosphorylation of the Thr-x-Tyr (T-x-Y) motif in the activation loop (T-loop). Four subgroups, designated A, B, C, and $\mathrm{D}$, have been identified based on phylogenetic analysis of MAPKs, and distinct functions in signal transduction within
\end{abstract}

Received for publication 12 May 2016. Accepted for publication 18 July 2016. This work was supported by grants from Shandong Provincial Natural Science Foundation, China (ZR2014CQ018); the Special Fund for Agro-scientific Research in the Public Interest, China (201203075); and the Special Fund for Shandong Provincial Fruit Innovative Team, China (SDAIT-03-022-04).

${ }^{1}$ Corresponding author. E-mails: qzliu001@126.com; xjzong812@163.com. cells in plants have been reported for each group (MAPK Group, 2002). Groups A-C, which possess a Thr-Glu-Tyr (T-E-Y) motif, have been studied in many plant species, including arabidopsis (Arabidopsis thaliana), tobacco (Nicotiana tabacum), and maize (Zea mays), and were reported to cause positive effects on stress tolerance during plant transformation with single genes under controlled conditions. For example, in arabidopsis, AtMPK3 and AtMPK6 from group A (Conroy et al., 2013; Droillard et al., 2002; Moon et al., 2003; Raghuram et al., 2015; Schikora et al., 2011; Wan et al., 2004) and AtMPK4 from group B (Ichimura et al., 2000, 2006; Roux et al., 2015) have been shown to play important roles in response to salt and cold stress and pathogen attacks. Ectopic expression of ZmSIMK1, a member of group B from maize, was reported to be involved in an improved tolerance to both biotic (e.g., Pseudomonas syringae) and abiotic stresses (e.g., salt and drought) in transgenic tobacco plants (Gu et al., 2010; Wang et al., 2014). Overexpression of another maize group A MAPK gene, ZmMPK5, in tobacco induced defense responses and conferred tolerance to salt and low-temperature stress in transgenic plants (Berberich et al., 1999; Zhang et al., 2014). These findings demonstrated the various roles of MAPK cascades in environmental stress responses and highlighted the potential application of MAPK genes for improving plant tolerance through biotechnology methods.

Because of the importance of MAPK cascades in plant resistance to biotic and abiotic stress, our goal was to identify and characterize MAPK genes in 'Gisela 6' to allow further work that would improve stress resistance in cherry rootstock through genetic engineering.

\section{Materials and Methods}

Plant material and treatment. In vitro plantlets of 'Gisela 6' were selected for qRT-PCR analysis. The plantlets 
were subcultured on Murashige and Skoog (MS) medium in the culture room held at $25^{\circ} \mathrm{C}$ with a $16 / 8$-h (light/dark) cycle for 6 weeks, and were then selected for different stress treatments. For osmotic and salinity stress challenges, in vitro plantlets of 'Gisela 6' were cultured in solid MS medium complemented with $200 \mathrm{mmol}$ mannitol or $200 \mathrm{mmol} \mathrm{NaCl}$, respectively. For the low-temperature treatment, the plantlets were cultured in solid MS medium in an artificial climate chest in which the temperature was held at $4{ }^{\circ} \mathrm{C}$ with a light/dark cycle of $16 / 8 \mathrm{~h}$. For the salicylic acid (SA) and methyl jasmonate (MeJA) treatments, the plantlets were cultured in solid MS medium complemented with $150 \mu \mathrm{mol}$ SA and $200 \mu \mathrm{mol} \mathrm{MeJA}$, respectively. For $P$. syringae pv. syringae infection, single bacterial isolates were cultured on King's B medium at $25^{\circ} \mathrm{C}$ for $48 \mathrm{~h}$. Before inoculation, bacterial cells were suspended in sterile tap water to produce a suspension containing $10^{8}-10^{9}$ colony-forming units $/ \mathrm{mL}$. The whole plantlets were inoculated by dipping in the bacterial suspension and the cocultivation occurred at $25^{\circ} \mathrm{C}$ with a $16 / 8$-h (light/dark) photoperiod. For all stress challenges, the whole in vitro plantlets were collected at $2,4,6,12,24,48$, and $72 \mathrm{~h}$, respectively, after treatment, immediately frozen in liquid nitrogen, and stored at $-80^{\circ} \mathrm{C}$ for RNA extraction. Plantlets cultured in the normal MS medium at $25^{\circ} \mathrm{C}$ with a $16 / 8$-h photoperiod served as the control for the qRT-PCR analysis. All samples included at least three independent biological repeats that were randomly selected from the vessels.

RNA EXTRACTION AND REVERSE TRANSCRIPTION. For total RNA extraction, frozen plantlets were crushed in liquid nitrogen with a mortar and pestle. RNA was extracted using the RN38-EASYspin Plus Plant RNA Kit (Aidlab, Beijing, China), in accordance with the manufacturer's instructions. The first strand complementary DNA (cDNA) was obtained using HiFiScript Quick gDNA Removal cDNA Kit (CWBIO, Beijing, China), according to the manufacturer's instructions.

Cloning ANd IDENTIFICATION OF MAPKS FROM THE CHERRY ROOTSTOCK 'Gisela 6'. A genome-wide identification of MAPK gene families in 'Gisela 6' rootstock was difficult due to the lack of genomic information for this cultivar. Thus, we searched and identified the MAPK candidate genes from a cDNA library of the 'Gisela 6' leaf samples based on the transcriptome sequencing, which was provided by a colleague in our group. Total RNA of the leaf samples taken from six different branches of two independent 5-year-old 'Gisela 6' trees was pooled before library preparation. The library preparations were sequenced by Novogene Bioinformatics Technology Co. (Beijing, China) on an Illumina HiSEq 2000 platform and 100-base pair (bp) paired end reads were generated. A total of 50,919 transcripts were assembled and obtained using the Trinity software (Grabherr et al., 2011). A number of MAPK candidate genes were identified from the de novo transcriptome assembly database by BLASTx search. Target sequences that were more than $1000 \mathrm{bp}$ in length, contained intact open reading frames (ORFs), and had greater than $60 \%$ homology to MAPK genes in other species were used as the templates for cDNA cloning. A total of 12 primer pairs were designed using Primer Premier 5.0 (Premier Biosoft, Palo Alto, CA) to amplify the full-length open reading frame of MAPK genes from cDNA and genomic DNA of 'Gisela 6' (Supplemental Table 1). All the amplified products were cloned into pEASY-Blunt Simple Cloning Vectors (Transgen, Beijing China) and sequenced (Sangon Biotech, Beijing, China). The sequences were designated as $P c M P K s$ and submitted to GenBank.

SEQUence ANALYsis of THE MAPKs. Multiple alignments of the deduced MAPK amino acid sequences were performed using the Clustal W program (Larkin et al., 2007). A phylogenetic tree was constructed using the MEGA 6 software (Tamura et al., 2013). Because no reference genome was available for cherry, the gene structure analyses of the PcMPKs were generated from the alignments of the cDNA and genomic DNA amplicon sequences.

REAL-TIME QRT-PCR ANALYSIS. Primers for real-time qRTPCR analysis were designed using Beacon Designer 8 software (Premier Biosoft) (Supplemental Table 1). Reactions were carried out on an ABI 7500 Fast Real-Time PCR System (Applied Biosystems, Foster City, CA) using the UltraSYBR mixture with Rox (CWBIO) following the manufacturer's instructions. The sweet cherry house-keeping gene $\beta$-actin (accession no. FJ560908) was selected as the internal control to calibrate the cDNA template of the corresponding samples. PCR conditions were as follows: an initial $95^{\circ} \mathrm{C}$ denaturation step for $10 \mathrm{~min}$, followed by 40 cycles of denaturation at $95^{\circ} \mathrm{C}$ for $15 \mathrm{~s}$ and annealing/extension at $55^{\circ} \mathrm{C}$ for $1 \mathrm{~min}$. The specificity of the PCR amplification procedure was determined with a heat-dissociation protocol after the final PCR cycle. Each

Table 1. The information of MAPK family genes in 'Gisela 6' cherry rootstock.

\begin{tabular}{|c|c|c|c|c|c|c|c|c|c|c|}
\hline \multirow[b]{2}{*}{ Name } & \multirow[b]{2}{*}{ Accession no. } & \multirow[b]{2}{*}{ ORF size (bp) } & \multirow[b]{2}{*}{ MAPK group } & \multirow[b]{2}{*}{ Homologs } & \multicolumn{6}{|c|}{ Regulated by } \\
\hline & & & & & Mannitol & $\mathrm{NaCl}$ & Low temp & SA & MeJA & Bacterium infection \\
\hline$\overline{P c M P K 3}$ & KT357359 & 1113 & $\mathrm{~A}$ & AtMPK3 & $\mathrm{Up}$ & Up & $\mathrm{Up}$ & Up & Up & $\mathrm{Up}$ \\
\hline РсMРК6 & KT357360 & 1224 & A & AtMPK6 & Up & Up & - & Up & - & Up \\
\hline PcMPK4-2 & KT357357 & 1122 & B & AtMPK4/11 & Up & $\mathrm{Up}$ & $\mathrm{Up}$ & $\mathrm{Up}$ & - & $\mathrm{Up}$ \\
\hline PcMPK13 & КT357358 & 1113 & $\mathrm{~B}$ & AtMPK13 & - & - & Up & - & - & - \\
\hline PcMPK1 & КT357361 & 1119 & $\mathrm{C}$ & AtMPK1/2 & Down & Down & - & Up & - & - \\
\hline РсMРК9-1 & КT357363 & 1281 & $\mathrm{D}$ & AtMPK9 & $\mathrm{Up}$ & Up & - & - & - & - \\
\hline PcMPK9-2 & КT357364 & 1881 & $\mathrm{D}$ & AtMPK9 & - & - & - & - & - & - \\
\hline PcMPK16 & KT357365 & 1683 & $\mathrm{D}$ & AtMPK16 & - & $\mathrm{Up}$ & Up & - & - & - \\
\hline РcMPK 20 & КT357366 & 1869 & $\mathrm{D}$ & AtMPK20 & $\mathrm{Up}$ & $\mathrm{Up}$ & $\mathrm{Up}$ & - & - & - \\
\hline PсMPK 18 & КT357367 & 1830 & D & AtMPK18/19 & Down & - & Down & - & Down & Down \\
\hline
\end{tabular}

$\mathrm{MAPK}=$ mitogen-activated protein kinase; MeJA = jasmonic acid methyl ester; ORF = open reading frame; $\mathrm{SA}=$ salicylic acid. 
measurement was carried out with at least three independent biological replicates, with PCR in triplicate to determine $\mathrm{Ct}$ values. Relative gene expression was analyzed according to the $2^{-\Delta \Delta \mathrm{Ct}}$ method. A two-sample $\mathrm{F}$ test was performed to compare two population variances. $P \leq 0.05$ indicated that significant difference of the variation of the gene expression could be deduced.

\section{Results}

IDENTIFICATION AND NOMENCLATURE OF MAPKS GENES IN 'Gisela 6'. A total of 12 putative $M A P K$ sequences with intact ORFs have been identified from the de novo transcriptome assembly of 'Gisela 6'. The sequence data were verified by PCR amplification from cDNA of 'Gisela 6'. The 12 target sequences met the following criteria: 1) significant homology with known MAPKs, 2) possessing 11 conserved subdomains in the amino acid sequence, and 3) possessing the activation loop with T-x-Y motif. Therefore, the target sequences were considered to be 'Gisela 6' MAPK genes. The nomenclature and the molecular characterization of the 12 novel MAPK genes from 'Gisela 6' are detailed in Table 1.

Multiple SEQuence AlignMent, Phylogenetic RELATIONSHIPS, AND GENE STRUCTURE ANALYSIS OF MAPK GENES IN 'GiSELA 6'. To explore the conserved and divergent amino acid sequences of 'Gisela 6' MAPKs, multiple sequence alignment was performed with the deduced amino acids sequences using Clustal W. The 12 novel PcMAPKs possessed the 11 conserved domains that are known to be characteristic of plant MAPKs. The activation loop containing a T-X-Y motif, which was considered the phosphorylation site for MAPK activity, was found between domains VII and VIII in all of the 12 PcMPKs. Seven PcMPKs carried a Thr-Glu-Tyr (T-E-Y) motif, whereas five PcMPKs carried a Thr-Asp-Tyr (T-D-Y) motif. The TDY domain-containing MPKs also had an extended C-terminal region, which is similar to MPKs from other plant species (Fig. 1).

To evaluate the evolutionary relationship of PcMPKs and other plant MAPKs, a phylogenetic tree was constructed using the MEGA 6 software with the neighbor-joining method. The
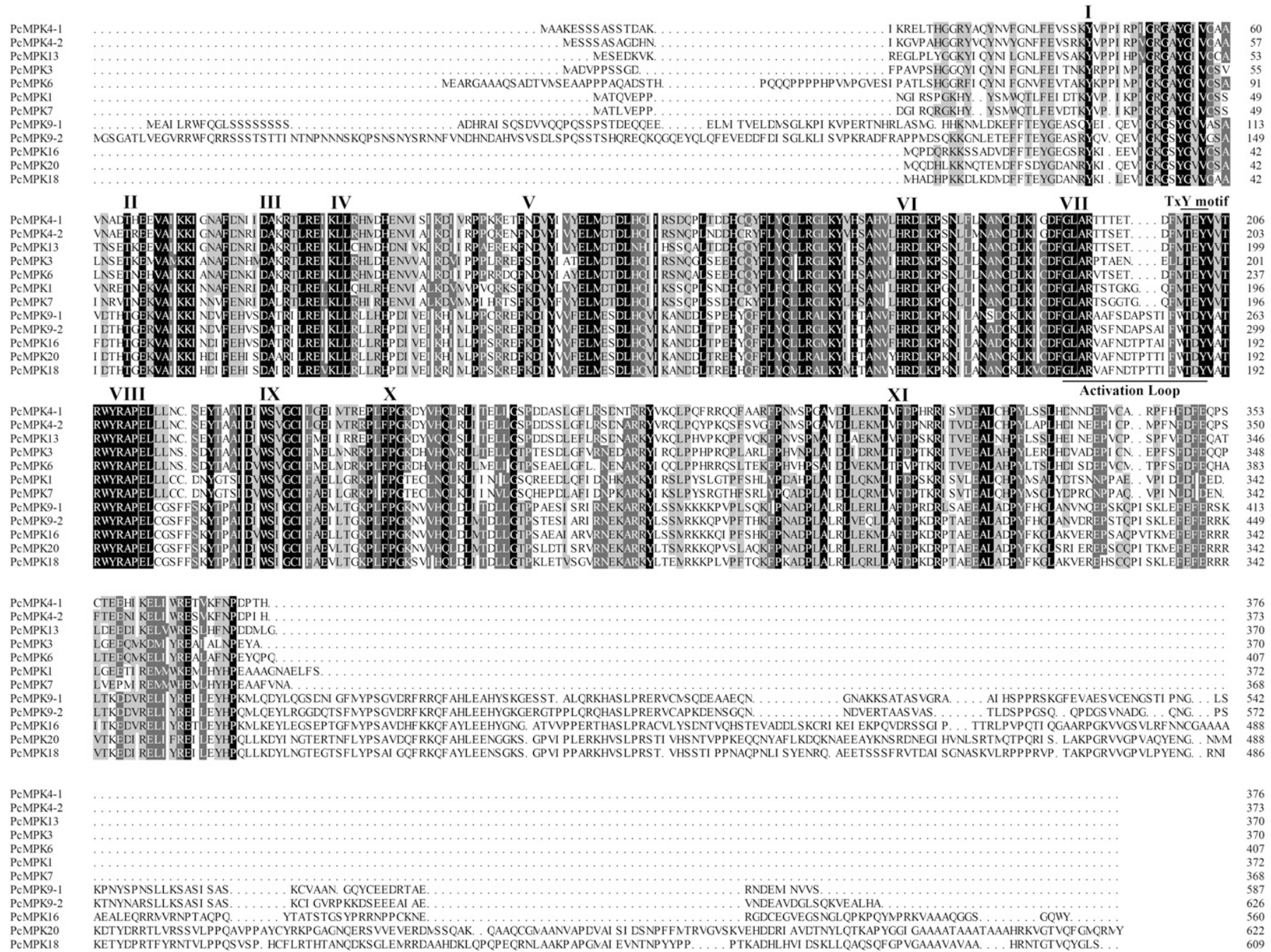

Fig. 1. Multiple alignment of PcMPKs amino acid sequences. The alignment was performed using the Clustal W program (Larkin et al., 2007). The characteristic regions containing 11 domains (I-XI), the activation loop, and Thr-x-Tyr (TXY) motif are presented in serine/threonine protein kinase and marked on the top of each row. The 12 novel PcMAPKs possessed the characteristic regions of plant mitogen-activated protein kinase (MAPKs). Seven PcMPKs carried a Thr-Glu-Tyr (T-E-Y) motif, whereas five PcMPKs carried a Thr-Asp-Tyr (TDY) motif. 
12 novel PcMPKs could be divided into four major groups (groups A-D). Among them, two PcMPKs were classified into each of group A and group $\mathrm{C}$, and three were classified into group B. Group D, which possessed a TDY motif, contained the most MAPKs homologs (five members) (Fig. 2); a similar distribution of MAPKs homologs has been observed in other plant species including arabidopsis and tobacco (MAPK Group, 2002; Zhang et al., 2013b). For example, eight of 20 AtMPKs were classified as group D in arabidopsis, and six of $17 \mathrm{NtMPKs}$ were classified as group D in tobacco. As mentioned above, PcMPKs in group D were notable for their TDY motif in the activation loop and an extended C-terminal region.

To understand duplication events within the MAPK gene family, full-length cDNA sequences were compared with the corresponding genomic DNA sequences to reveal the exon/ intron structures of each $P c M P K$ gene. The number of introns varied from one (group $\mathrm{C}$ members) to nine (PcMPK16 from group D) (Fig. 3). Previous studies showed that most genes within the same subfamily shared a similar exon/intron structure (Cakir and Kilickaya, 2015; MAPK Group, 2002; Zhang et al., 2013a, 2013b). Similar results were observed here. For example, $P c M P K 1$ and $P c M P K 7$ from group $\mathrm{C}$ possessed only one intron, whereas PcMPK4-1, PcMPK4-2, and PcMPK13 from group $\mathrm{B}$, each contained five introns. However, the length of the introns and exons were quite different even between members of the same group. Some modest differences in organization were observed between PcMPK3 and PcMPK6, both of which were classified into group A. For example, PcMPK3 contained five introns, whereas PcMPK6 contained four introns. PcMPKs from group D included the most complex gene structures, containing eight or nine introns and possessing large differences in the length of genomic sequences between group members.

EXPRESSION PROFILES OF PCMPKS IN RESPONSE TO STRESS STIMULI, SIGNAL MOLECULES, AND PATHOGEN INFECTION. To identify the candidate genes potentially involved in responses to abiotic or biotic stresses, we analyzed the expression profiles of PcMPKs following exposure to environmental stress (osmotic, salinity, or low temperature), exposure to defense signaling molecules (SA and MeJA), and pathogen infection (P. syringae pv. syringae).

A total of eight $P c M P K$ genes exhibited changes in expression following mannitol treatment (Fig. 4A). Among them, five PcMPKs (PcMPK4-2/3/6/9-1/20) were upregulated, with PcMPK4-2, PcMPK3, and PcMPK6 being especially highly induced at 6 to $12 \mathrm{~h}$ after treatment. The remaining three genes (PcMPK1/7/18) showed downregulated expression at different levels under mannitol treatment.

Following exposure to $\mathrm{NaCl}$ treatment, eight PcMPKs (PcMPK4-1/4-2/3/6/7/9-1/16/20) showed significant changes in expression (Fig. 4B). Inductive effects caused by $\mathrm{NaCl}$ showed different patterns across genes, with either an initial increase followed by a slight decrease (e.g., PcMPK7 and $P c M P K 20$ ) or increased levels that were maintained throughout the treatment (e.g., PcMPK4-1/4-2/3/6). PcMPK1 displayed an obvious downregulation following $\mathrm{NaCl}$ treatment; similar suppression of gene expression was also observed during treatment with mannitol (Fig. 4A).

Seven PcMPKs (PcMPK 4-1/4-2/13/3/7/16/20) were induced by low temperature (Fig. 4C). The expressions of PCMPK13, PcMPK3, PCMPK16, and PCMPK20 were increased significantly and maintained at a relatively high level

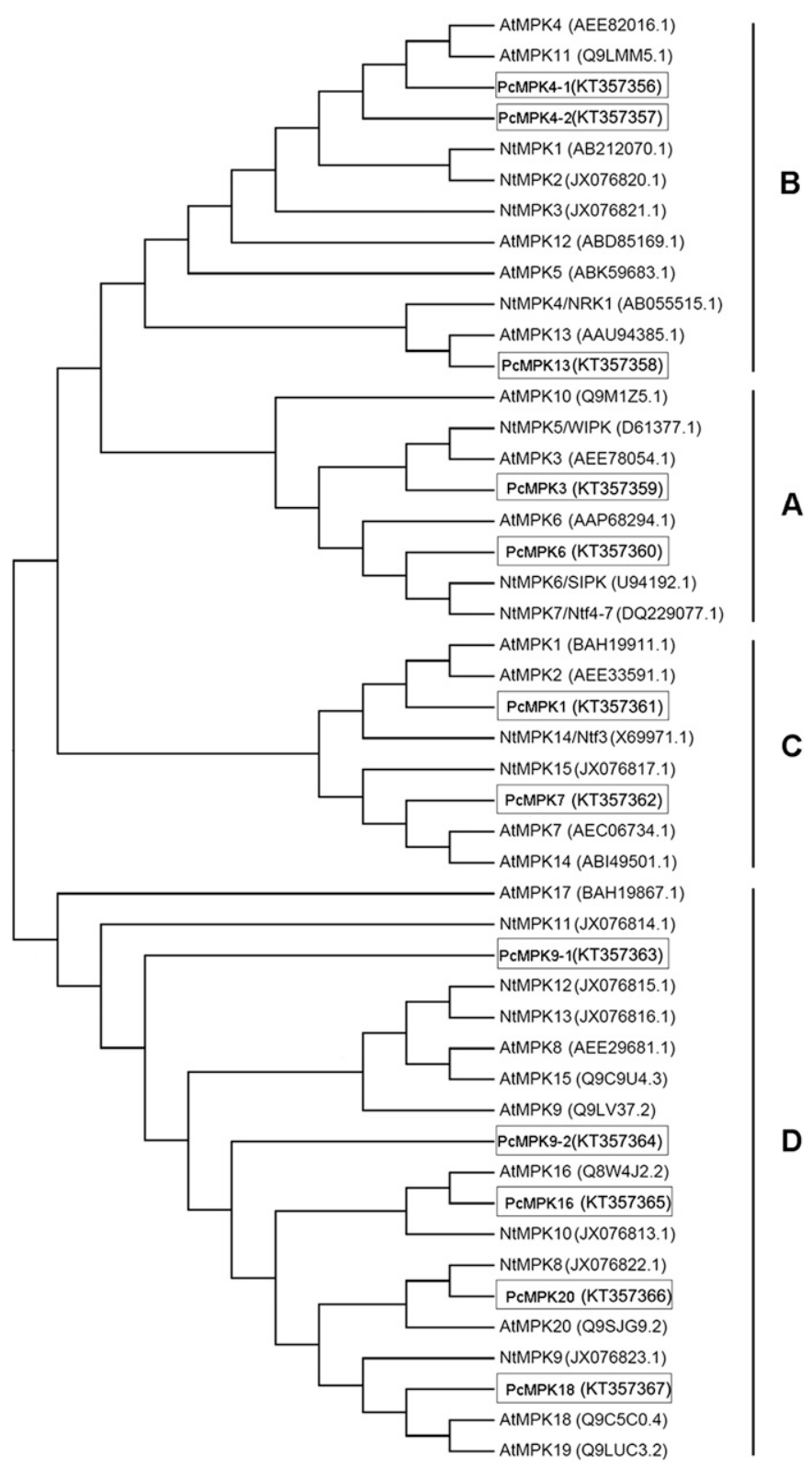

Fig. 2. Phylogenetic analysis of the PcMPKs. A phylogenetic tree was constructed based on the complete protein sequence alignment of mitogenactivated protein kinase (MAPKs) in arabidopsis, tobacco, and 'Gisela 6' cherry rootstock using the neighbor-joining method with bootstrapping analysis. Different gene clusters are denoted as A-D. The subgroups are marked by the black lines. Among the 12 novel PcMPKs, two were classified into each of group A and group C, and three were classified into group B. Group D contained the most MAPKs homologs (five members).

during the entire temperature experiment. PcMPK4-1, PcMPK4-2, and PcMPK7 were moderately induced. The expression of $P c M P K 18$ was suppressed immediately after treatment and then recovered slowly to the control levels.

To explore whether MAPK cascades were responsive to $P$. syringae pv. syringae infection, we inoculated 'Gisela 6' plantlets with $P$. syringae pv. syringae and analyzed the expression profiles of PcMPKs. PcMPK4-1 was greatly induced when plants were infected with the pathogen (Fig. 4D). The expression of PcMPK4-2, PcMPK3, and PcMPK6 were also stimulated substantially after pathogen exposure. In 


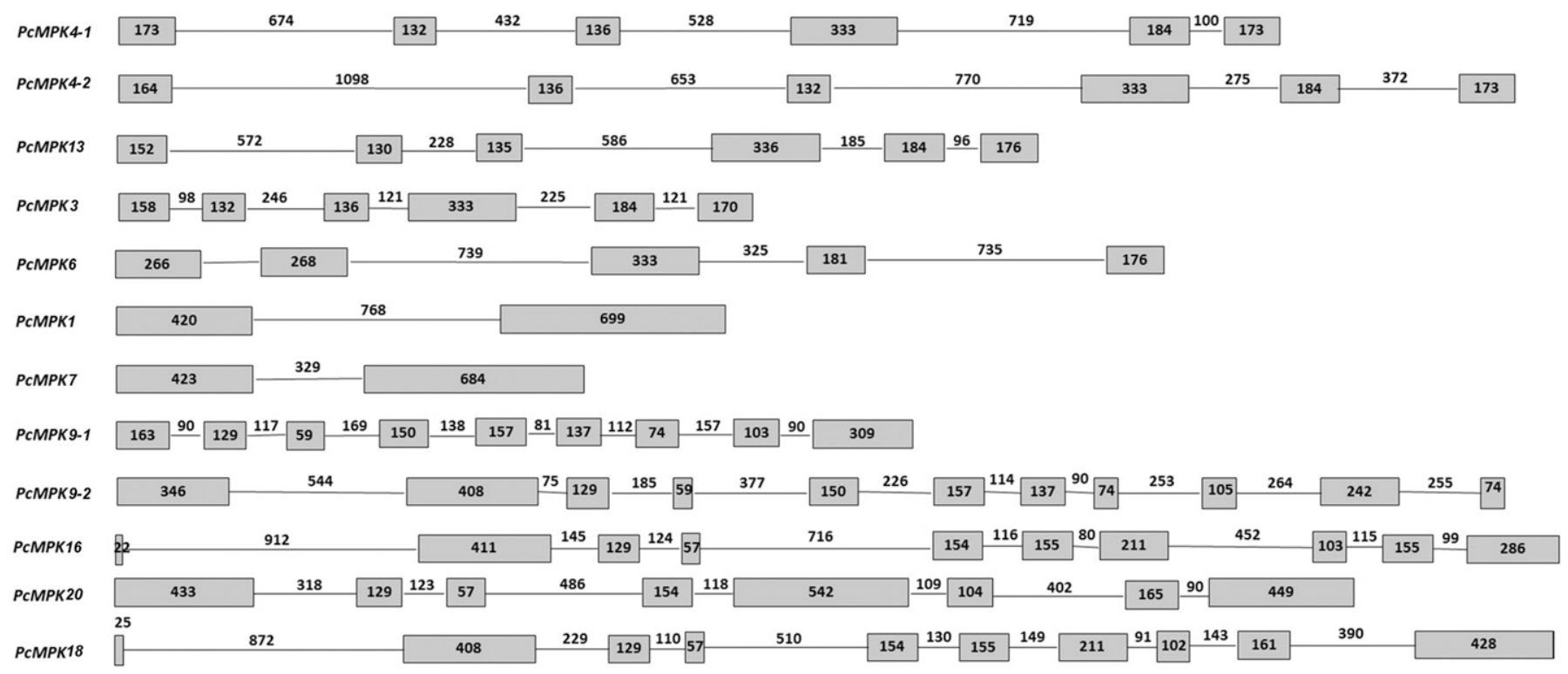

Fig. 3. The exon/intron structure of 'Gisela 6' cherry rootstock PcMPKs genes. Exons and introns of the genes are indicated by gray boxes and black lines, respectively. The size of exons and introns is given inside the boxes and on top of the lines. Among the 12 novel PcMPKs, the number of introns varied from one (group C members) to nine (PcMPK16 from group D). Most members within the same subgroup shared a similar exon/intron structure.

contrast, the expression of $P c M P K 18$ was sharply suppressed after pathogen infection, and the expression of PcMPK13 was also slightly decreased.

SA and MeJA are two important signaling molecules involved in plant defense against pathogen infection. To explore more candidate loci implicated in response to potential biotic stress, we analyzed the expression profiles of PcMPKs treated with SA and MeJA (Fig. 4E-F). PcMPK4-1, PcMPK4-2, $P c M P K 3, P c M P K 6$, and PcMPK1 were upregulated 2- to 4-fold over the controls under SA treatment. PcMPK3 was also induced significantly by MeJA. However, the expression of PcMPK13 and PcMPK18 were downregulated when treated with SA, with a mild recovery $24 \mathrm{~h}$ after treatment. These two genes were also downregulated slightly by MeJA. Notably, both of them were suppressed by $P$. syringae pv. syringae infection, as shown in Fig. 4D. These results indicate that $P c M P K 13$ and PcMPK18 may respond to pathogen infection in a different way from those candidates that were upregulated, such as PcMPK3 and PcMPK6.

\section{Discussion}

The engineered MAPK cascades were proposed to be the potential application for improving plant stress tolerance (Moustafa, 2014a, 2014b). To date, a conserved number of MAPK families have been identified and functionally characterized in arabidopsis, tobacco, grape (Vitis vinifera), apple (Malus domestica), etc. (Cakir and Kilickaya, 2015; MAPK Group, 2002; Zhang et al., 2013a, 2013b). However, no data on MAPK were available for either sweet cherry or close-related species. A genome-wide identification of MAPK genes family in 'Gisela 6' rootstock was difficult due to the lack of genomic information for this cultivar. Herein, a total of 12 novel MAPKs were isolated and analyzed from cherry rootstock 'Gisela 6'. Although we could not identify MAPK families at a genomewide scale in this study, the whole genome assembly for peach (Prunus persica) may offer some hints for analysis. A total of
$15 M A P K$ candidates were identified from the released phytozome, which included the gene annotation of $225.7 \mathrm{Mb}$ arranged in eight chromosomes (Verde et al., 2013). Therefore, due to the conserved number of MAPKs in other plants, it is likely that we have identified most, if not all, of the MAPKs in 'Gisela 6'.

Phylogenetic relationship analysis showed that PcMPK3 and PcMPK6 were homologs of AtMPK3 and AtMPK6, which are the most extensively studied MAPKs in arabidopsis, and are involved in plant resistance (Conroy et al., 2013; Droillard et al., 2002; Moon et al., 2003; Raghuram et al., 2015; Schikora et al., 2011; Wan et al., 2004). Their homologs in tobacco, WIPK and SIPK, have also been shown in many studies to regulate response to abiotic and biotic stresses (Kallenbach et al., 2010; Katou et al., 2005; Kim et al., 2011; Kobayashi et al., 2010; Sharma et al., 2003; Zhang and Klessig, 1998). AtMPK4, another well-studied MAPK in arabidopsis, was associated with response to low temperature, drought, wounding injury, and pathogen infection (Ichimura et al., 2006; Roux et al., 2015). As shown in Fig. 2, PcMPK4-1 and PcMPK4-2 were clustered into the same subgroup as AtMPK4 in the phylogenetic tree. Because MAPK genes with the same function typically cluster into the same subgroup, the phylogenetic analysis may provide a basis for exploring the functions of PcMPKs. It is reasonable to speculate that PcMPK3, PcMPK6, PcMPK4-1, and PcMPK4-2 may be involved in stress responses in 'Gisela 6'.

In addition, this study reported the intron-exon structures of $P c M P K$ genes. Because no genomic sequences are currently available, we identified and sequenced each $P c M P K$ gene from plant total cDNA and genomic DNA separately and aligned these sequences to determine the intron-exon structure for each gene. The purpose of the structure analysis was not only to compare the intron-exon splicing with that in other plant species, but also to provide sequence references for the identification of the regulatory regions of genes with important functions in stress resistance. 
A
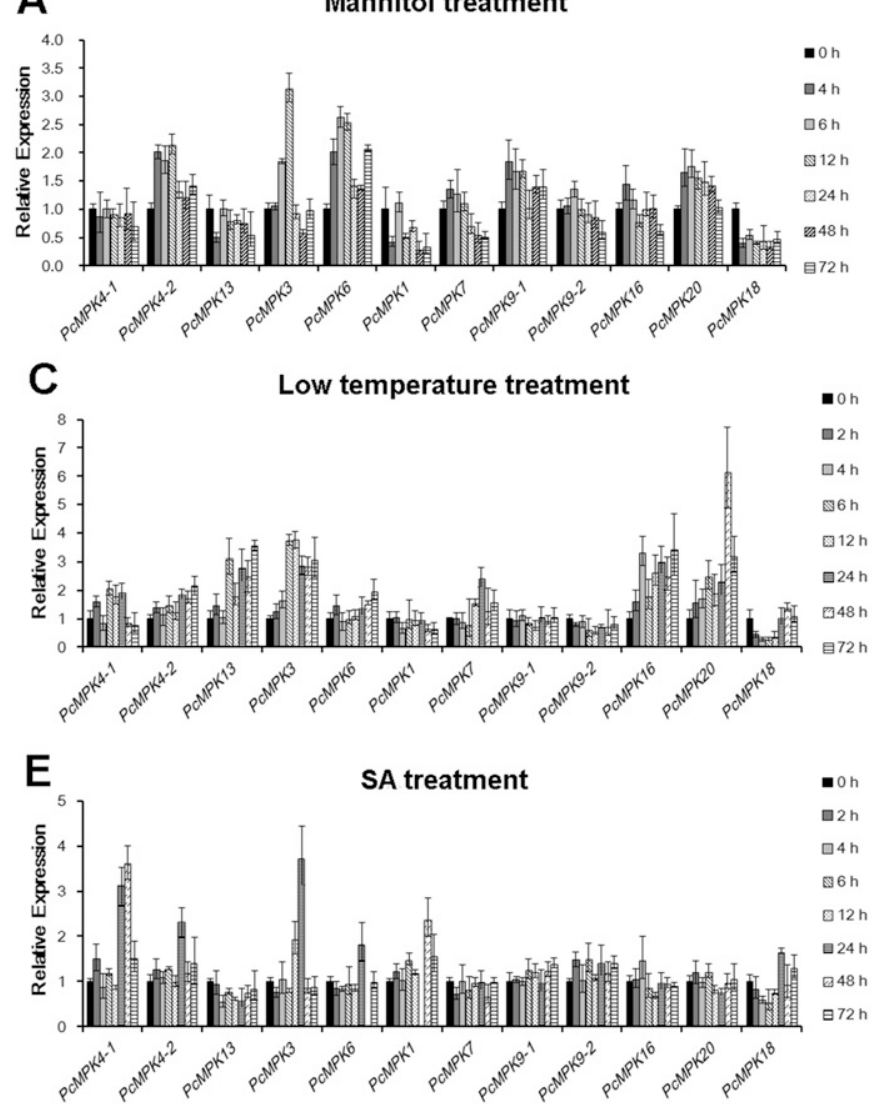

B
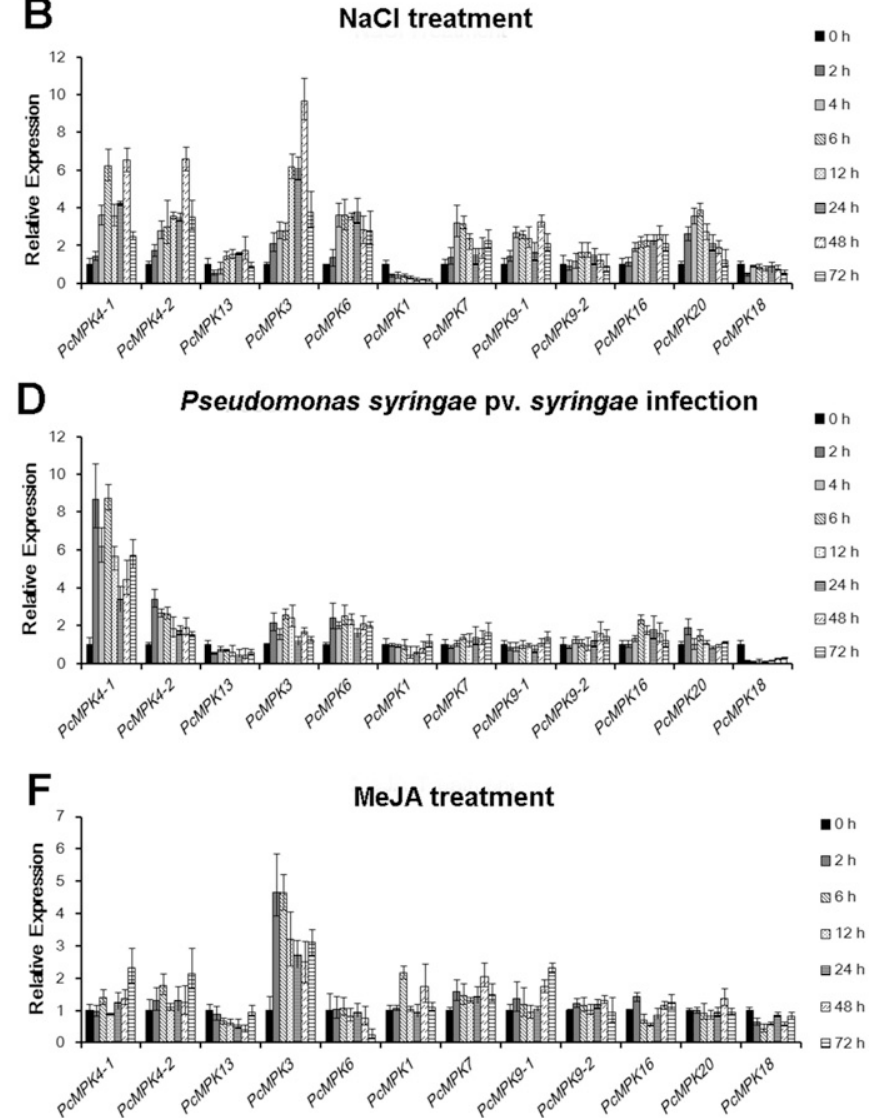

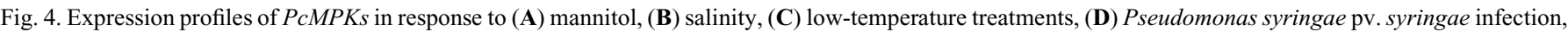
(E) salicylic acid, and (F) jasmonic acid methyl ester stimuli. Quantitative real-time reverse transcription polymerase chain reaction (qRT-PCR) was used to detect the mRNA levels of PcMPKs under the experimental treatments. The constitutive gene $\beta$-actin was used as a reference. Each measurement was carried out with at least three independent biological replicates, with PCR in triplicate to determine $\mathrm{Ct}$ values. Relative gene expression was analyzed according to the $2^{-\Delta \Delta \mathrm{Ct}}$ method. Error bars represent the SE of at least three independent biological replicates.

Because gene expression patterns can provide important clues to gene function, we conducted real-time qRT-PCR analysis to reveal the expression profiles of the 12 PcMPK genes in response to several environmental stresses, signaling molecules, and potential pathogen infection. In our study, $P c M P K 3$ and PcMPK6 were significantly induced by most of the treatments, including exposure to mannitol, $\mathrm{NaCl}$, a bacterial pathogen, SA and MeJA (Fig. 4). Similar expression profiles were found in their orthologs AtMPK3/AtMPK6 in arabidopsis. AtMPK3 has been shown to be particularly strongly associated with numerous abiotic and biotic stress responses in arabidopsis. For example, AtMPK3/AtMPK6 and their upstream kinase AtMKK4/AtMKK5 were activated by various biotic and abiotic stimuli (Colcombet and Hirt, 2008; Pitzschke et al., 2009; Rodriguez et al., 2010; Samajova et al., 2013; Sinha et al., 2011). Both AtMPK3/6 and AtMKK4/5 have overlapping functions. Mutant $A t M K K 4$ s that were defective in the AtMPK3/6-upstream regulatory MAPK kinase were less tolerant to osmotic stress. In contrast, AtMPK $3 / 6$ hyperactivation via AtMKK4 overexpression enhanced stress tolerance (Kim et al., 2011). Given the expression profiles of PcMPK3/6 in response to stress and the previous reports of orthologs in other plant species, it was reasonable to speculate that these two MAPK members may be involved in the cross-talk node of different signaling transduction pathways. PcMPK3 and
PcMPK6 could act as a potential "regulating switch" during rewiring of MAPK transgenic circuits to improve plant stress tolerance.

In addition, PcMPK4-1 and PcMPK4-2 were also induced by $\mathrm{NaCl}$, low temperature, or bacterial infection. Similar characteristics were found for AtMPK4, the ortholog of PcMPK4-1/4-2 in arabidopsis. AtMPK4 was reported to be activated by low temperature, low humidity, hyperosmolarity, and wounding (Ichimura et al., 2000); AtMPK4 also appears to play a role in negatively regulating immune responses (Berriri et al., 2012; Gao et al., 2008; Kong et al., 2012) and in hyperosmolarity resistance (Droillard et al., 2004) in arabidopsis. These studies suggest that PcMPK4-1 and PcMPK4-2 may play a potential role in plant defensive responses and resistance to osmotic stress or other environmental stresses.

Finally, PcMPK18 was especially interesting due to its significant negative expression profile in response to stress treatments. As shown in Fig. 4, the expression of PcMPK18 was sharply suppressed throughout the mannitol treatment. When treated with low temperature, the gene expression level decreased rapidly, but recovered slowly over the $24 \mathrm{~h}$ after treatment. Pseudomonas syringae pv. syringae was reported to induce a number of important diseases in Prunus species. It caused a variety of symptoms in host plants, such as flower blast, dead dormant buds, shoot-tip dieback, and stem cankers 
(Qiu et al., 2008; Spotts and Cervantes, 1995). Infection with $P$. syringae pv. syringae also caused significant suppression of PcMPK18. The transcripts of $P C M P K 18$ were rapidly reduced to $\approx 1 / 10$ of that in control samples $2 \mathrm{~h}$ after treatment. Exogenous SA molecules may also suppress the expression of PcMPK18 at the beginning of treatment. However, expression increased slightly $24 \mathrm{~h}$ after the signaling molecule application. Although group D MAPKs were notable for their TDY motif and extended C-terminal sequences, no additional details about gene function could be obtained in the present study. Thus, it will be interesting to explore the potential roles that PcMPK18 plays in stress resistance.

Herein, we presented a comprehensive analysis of 12 novel MAPK genes in the cherry rootstock 'Gisela 6', with an emphasis on the potential functional characterization of stress responses. PcMPK4-1, PcMPK4-2, PcMPK3, PcMPK6, and $P c M P K 18$ were revealed to be potentially involved in plant resistance to environmental stress. This study may provide the basis for further analysis on the physiological functions of $P c M P K s$ in environmental tolerance in cherry rootstocks.

\section{Literature Cited}

Berberich, T., H. Sano, and T. Kusano. 1999. Involvement of a MAP kinase, $Z m M P K 5$, in senescence and recovery from low-temperature stress in maize. Mol. Gen. Genet. 262:534-542.

Berriri, S., A.V. Garcia, N. Frei dit Frey, W. Rozhon, S. Pateyron, N. Leonhardt, J.L. Montillet, J. Leung, H. Hirt, and J. Colcombet. 2012. Constitutively active mitogen-activated protein kinase versions reveal functions of Arabidopsis MPK4 in pathogen defense signaling. Plant Cell 24:4281-4293.

Cakir, B. and O. Kilickaya. 2015. Mitogen-activated protein kinase cascades in Vitis vinifera. Front. Plant Sci. 6:556.

Colcombet, J. and H. Hirt. 2008. Arabidopsis MAPKs: A complex signalling network involved in multiple biological processes. Biochem. J. 413:217-226.

Conroy, C., J. Ching, Y. Gao, X. Wang, C. Rampitsch, and T. Xing. 2013. Knockout of AtMKK1 enhances salt tolerance and modifies metabolic activities in Arabidopsis. Plant Signal. Behav. 8:e24206.

Droillard, M.J., M. Boudsocq, H. Barbier-Brygoo, and C. Lauriere. 2004. Involvement of MPK4 in osmotic stress response pathways in cell suspensions and plantlets of Arabidopsis thaliana: Activation by hypoosmolarity and negative role in hyperosmolarity tolerance. FEBS Lett. 574:42-48.

Droillard, M., M. Boudsocq, H. Barbier-Brygoo, and C. Lauriere. 2002. Different protein kinase families are activated by osmotic stresses in Arabidopsis thaliana cell suspensions. Involvement of the MAP kinases AtMPK3 and AtMPK6. FEBS Lett. 527:43-50.

Gao, M., J. Liu, D. Bi, Z. Zhang, F. Cheng, S. Chen, and Y. Zhang. 2008. MEKK1, MKK1/MKK2 and MPK4 function together in a mitogen-activated protein kinase cascade to regulate innate immunity in plants. Cell Res. 18:1190-1198.

Grabherr, M.G., B.J. Haas, M. Yassour, J.Z. Levin, D.A. Thompson, I. Amit, X. Adiconis, L. Fan, R. Raychowdhury, Q. Zeng, Z. Chen, E. Mauceli, N. Hacohen, A. Gnirke, N. Rhind, F. di Palma, B.W. Birren, C. Nusbaum, K. Lindblad-Toh, N. Friedman, and A. Regev. 2011. Full-length transcriptome assembly from RNA-seq data without a reference genome. Nat. Biotechnol. 29:644-652.

Gu, L., Y. Liu, X. Zong, L. Liu, D.P. Li, and D.Q. Li. 2010. Overexpression of maize mitogen-activated protein kinase gene, ZmSIMK1 in Arabidopsis increases tolerance to salt stress. Mol. Biol. Rpt. 37:4067-4073.

Ichimura, K., C. Casais, S.C. Peck, K. Shinozaki, and K. Shirasu. 2006. MEKK1 is required for MPK4 activation and regulates tissuespecific and temperature-dependent cell death in Arabidopsis. J. Biol. Chem. 281:36969-36976.
Ichimura, K., T. Mizoguchi, R. Yoshida, T. Yuasa, and K. Shinozaki. 2000. Various abiotic stresses rapidly activate Arabidopsis MAP kinases ATMPK4 and ATMPK6. Plant J. 24:655-665.

Kallenbach, M., F. Alagna, I.T. Baldwin, and G. Bonaventure. 2010. Nicotiana attenuata SIPK, WIPK, NPR1, and fatty acid-amino acid conjugates participate in the induction of jasmonic acid biosynthesis by affecting early enzymatic steps in the pathway. Plant Physiol. 152:96-106.

Katou, S., H. Yoshioka, K. Kawakita, O. Rowland, J.D. Jones, H. Mori, and N. Doke. 2005. Involvement of PPS3 phosphorylated by elicitorresponsive mitogen-activated protein kinases in the regulation of plant cell death. Plant Physiol. 139:1914-1926.

Kim, S.H., D.H. Woo, J.M. Kim, S.Y. Lee, W.S. Chung, and Y.H. Moon. 2011. Arabidopsis MKK4 mediates osmotic-stress response via its regulation of MPK3 activity. Biochem. Biophys. Res. Commun. 412:150-154.

Kobayashi, M., S. Seo, K. Hirai, A. Yamamoto-Katou, S. Katou, H. Seto, T. Meshi, I. Mitsuhara, and Y. Ohashi. 2010. Silencing of $W I P K$ and SIPK mitogen-activated protein kinases reduces tobacco mosaic virus accumulation but permits systemic viral movement in tobacco possessing the $N$ resistance gene. Mol. Plant Microbe Interact. 23:1032-1041.

Koc, A., Z. Celik, M. Akbulut, S. Bilgener, S. Ercisli, M. Gunes, R. Gercekcioglu, and A. Esitken. 2013. Morphological characterization of cherry rootstock candidates selected from Central and East Black Sea Regions in Turkey. Sci. World J. 2013:916520.

Kong, Q., N. Qu, M. Gao, Z. Zhang, X. Ding, F. Yang, Y. Li, O.X. Dong, S. Chen, X. Li, and Y. Zhang. 2012. The MEKK1-MKK1/ MKK2-MPK4 kinase cascade negatively regulates immunity mediated by a mitogen-activated protein kinase kinase kinase in Arabidopsis. Plant Cell 24:2225-2236.

Larkin, M.A., G. Blackshields, N.P. Brown, R. Chenna, P.A. McGettigan, H. McWilliam, F. Valentin, I.M. Wallace, A. Wilm, R. Lopez, J.D. Thompson, T.J. Gibson, and D.G. Higgins. 2007. Clustal W and Clustal $\mathrm{X}$ version 2.0. Bioinformatics 23:2947-2948.

Ligterink, W. 2000. MAP kinases in plant signal transduction: How many, and what for? Results Probl. Cell Differ. 27:11-27.

Ligterink, W. and H. Hirt. 2001. Mitogen-activated protein MAP kinase pathways in plants: Versatile signaling tools. Intl. Rev. Cytol. 201:209-275.

MAPK Group. 2002. Mitogen-activated protein kinase cascades in plants: A new nomenclature. Trends Plant Sci. 7:301-308.

Mizoguchi, T., K. Ichimura, R. Yoshida, and K. Shinozaki. 2000. MAP kinase cascades in Arabidopsis: Their roles in stress and hormone responses. Results Probl. Cell Differ. 27:29-38.

Moon, H., B. Lee, G. Choi, D. Shin, D.T. Prasad, O. Lee, S.S. Kwak, D.H. Kim, J. Nam, J. Bahk, J.C. Hong, S.Y. Lee, M.J. Cho, C.O. Lim, and D.J. Yun. 2003. NDP kinase 2 interacts with two oxidative stress-activated MAPKs to regulate cellular redox state and enhances multiple stress tolerance in transgenic plants. Proc. Natl. Acad. Sci. USA 100:358-363.

Moustafa, K. 2014a. Improving plant stress tolerance: Potential applications of engineered MAPK cascades. Trends Biotechnol. 32:389-390.

Moustafa, K. 2014b. MAPK transgenic circuit to improve plant stresstolerance? Plant Signal. Behav. 9:e970101.

Nakagami, H., A. Pitzschke, and H. Hirt. 2005. Emerging MAP kinase pathways in plant stress signalling. Trends Plant Sci. 10:339-346.

Pitzschke, A., A. Schikora, and H. Hirt. 2009. MAPK cascade signalling networks in plant defence. Curr. Opin. Plant Biol. 12:421-426.

Qiu, W., F. Xu, G. Xie, L. Xu, Y. Huai, B. Li, S. Yu, and J. Qian. 2008. Identification of Pseudomonas syringae pv. syringae causing pear blossom and bud blast in China. Scientia Agricultura Sinica 41:2657-2662.

Raghuram, B., A.H. Sheikh, Y. Rustagi, and A.K. Sinha. 2015. MicroRNA biogenesis factor DRB1 is a phosphorylation target of mitogen activated protein kinase MPK3 in both rice and Arabidopsis. FEBS J. 282:521-536. 
Rodriguez, M.C., M. Petersen, and J. Mundy. 2010. Mitogen-activated protein kinase signaling in plants. Annu. Rev. Plant Biol. 61:621649.

Roux, M.E., M.W. Rasmussen, K. Palma, S. Lolle, A.M. Regue, G. Bethke, J. Glazebrook, W. Zhang, L. Sieburth, M.R. Larsen, J. Mundy, and M. Petersen. 2015. The mRNA decay factor PAT1 functions in a pathway including MAP kinase 4 and immune receptor SUMM2. EMBO J. 34:593-608.

Samajova, O., O. Plihal, M. Al-Yousif, H. Hirt, and J. Samaj. 2013. Improvement of stress tolerance in plants by genetic manipulation of mitogen-activated protein kinases. Biotechnol. Adv. 31:118-128.

Schikora, A., S.T. Schenk, E. Stein, A. Molitor, A. Zuccaro, and K.H. Kogel. 2011. N-acyl-homoserine lactone confers resistance toward biotrophic and hemibiotrophic pathogens via altered activation of AtMPK6. Plant Physiol. 157:1407-1418.

Sharma, P.C., A. Ito, T. Shimizu, R. Terauchi, S. Kamoun, and H. Saitoh. 2003. Virus-induced silencing of WIPK and SIPK genes reduces resistance to a bacterial pathogen, but has no effect on the INF1-induced hypersensitive response (HR) in Nicotiana benthamiana. Mol. Genet. Genomics 269:583-591.

Sinha, A.K., M. Jaggi, B. Raghuram, and N. Tuteja. 2011. Mitogenactivated protein kinase signaling in plants under abiotic stress. Plant Signal. Behav. 6:196-203.

Song, G.Q. 2014. Recent advances and opportunities in cherry biotechnology. Acta Hort. 1020:89-97.

Song, G.Q. and K.C. Sink. 2006. Transformation of Montmorency sour cherry (Prunus cerasus L.) and Gisela 6 (P. cerasus $\times P$. canescens $)$ cherry rootstock mediated by Agrobacterium tumefaciens. Plant Cell Rpt. 25:117-123.

Song, G.Q., K.C. Sink, A.E. Walworth, M.A. Cook, R.F. Allison, and G.A. Lang. 2013. Engineering cherry rootstocks with resistance to Prunus necrotic ring spot virus through RNAi-mediated silencing. Plant Biotechnol. J. 11:702-708.

Spotts, R.A. and L.A. Cervantes. 1995. Factors affecting the severity of bacterial canker of pear caused by Pseudomonas syringae pv. syringae. Plant Pathol. 44:325-331.

Tamura, K., G. Stecher, D. Peterson, A. Filipski, and S. Kumar. 2013. MEGA6: Molecular evolutionary genetics analysis version 6.0. Mol. Biol. Evol. 30:2725-2729.

Usenik, V., N. Fajt, M. Mikulic-Petkovsek, A. Slatnar, F. Stampar, and R. Veberic. 2010. Sweet cherry pomological and biochemical characteristics influenced by rootstock. J. Agr. Food Chem. 58:4928-4933.
Verde, I., A.G. Abbott, S. Scalabrin, S. Jung, S. Shu, F. Marroni, T. Zhebentyayeva, M.T. Dettori, J. Grimwood, F. Cattonaro, A. Zuccolo, L. Rossini, J. Jenkins, E. Vendramin, L.A. Meisel, V. Decroocq, B. Sosinski, S. Prochnik, T. Mitros, A. Policriti, G. Cipriani, L. Dondini, S. Ficklin, D.M. Goodstein, P. Xuan, C. Del Fabbro, V. Aramini, D. Copetti, S. Gonzalez, D.S. Horner, R. Falchi, S. Lucas, E. Mica, J. Maldonado, B. Lazzari, D. Bielenberg, R. Pirona, M. Miculan, A. Barakat, R. Testolin, A. Stella, S. Tartarini, P. Tonutti, P. Arus, A. Orellana, C. Wells, D. Main, G. Vizzotto, H. Silva, F. Salamini, J. Schmutz, M. Morgante, and D.S. Rokhsar. 2013. The high-quality draft genome of peach (Prunus persica) identifies unique patterns of genetic diversity, domestication and genome evolution. Nat. Genet. 45:487-494.

Wan, J., S. Zhang, and G. Stacey. 2004. Activation of a mitogenactivated protein kinase pathway in arabidopsis by chitin. Mol. Plant Pathol. 5:125-135.

Wang, L., Y. Liu, G. Cai, S. Jiang, J. Pan, and D. Li. 2014. Ectopic expression of ZmSIMK1 leads to improved drought tolerance and activation of systematic acquired resistance in transgenic tobacco. J. Biotechnol. 172:18-29.

Zhang, D., S. Jiang, J. Pan, X. Kong, Y. Zhou, Y. Liu, and D. Li. 2014. The overexpression of a maize mitogen-activated protein kinase gene (ZmMPK5) confers salt stress tolerance and induces defence responses in tobacco. Plant Biol. (Stuttg.) 16:558-570.

Zhang, S. and D.F. Klessig. 1998. Resistance gene $N$-mediated de novo synthesis and activation of a tobacco mitogen-activated protein kinase by tobacco mosaic virus infection. Proc. Natl. Acad. Sci. USA 95:7433-7438.

Zhang, S., R. Xu, X. Luo, Z. Jiang, and H. Shu. 2013a. Genome-wide identification and expression analysis of MAPK and MAPKK gene family in Malus domestica. Gene 531:377-387.

Zhang, T., Y. Liu, T. Yang, L. Zhang, S. Xu, L. Xue, and L. An. 2006. Diverse signals converge at MAPK cascades in plant. Plant Physiol. Biochem. 44:274-283.

Zhang, X., T. Cheng, G. Wang, Y. Yan, and Q. Xia. 2013b. Cloning and evolutionary analysis of tobacco MAPK gene family. Mol. Biol. Rpt. 40:1407-1415.

Zhao, D. and G.Q. Song. 2014. Rootstock-to-scion transfer of transgene-derived small interfering RNAs and their effect on virus resistance in nontransgenic sweet cherry. Plant Biotechnol. J. 12:1319-1328.

Zwerger, K. and H. Hirt. 2001. Recent advances in plant MAP kinase signalling. Biol. Chem. 382:1123-1131. 
Supplemental Table 1. Sequences of primers used for PCR amplification and qRT-PCR analysis of PcMPKs.

\begin{tabular}{|c|c|}
\hline Gene name & Primer sequences \\
\hline \multicolumn{2}{|r|}{ Primers for gene amplification of $P c M P K S$} \\
\hline \multirow[t]{2}{*}{ PcMPK4-1 } & Fwd: TCGAGAATCACGGGCACCAT \\
\hline & Rev: ATCTACAGCCCAAACAAACG \\
\hline \multirow[t]{2}{*}{ PcMPK4-2 } & Fwd: TATTCTCCTCCATCATTAGCG \\
\hline & Rev: AATCCAATCACTCCAGCACA \\
\hline \multirow[t]{2}{*}{ PcMPK13 } & Fwd: TCCACCAGCTTTTCTAAATT \\
\hline & Rev: GAAATTAGATCGCTGCTGAC \\
\hline \multirow[t]{2}{*}{ PcMPK3 } & Fwd: ACGGACTTGCACAGTGTATT \\
\hline & Rev: CATAGGTATAGAAACCGAGTA \\
\hline \multirow[t]{2}{*}{ РсMРК6 } & Fwd: TTCTCACTGCCATTACTACAA \\
\hline & Rev: ACCAAACAAACGGAACATAG \\
\hline \multirow[t]{2}{*}{ PcMPK1 } & Fwd: TAGGGCTTGCGTCTGTGAAT \\
\hline & Rev: GCTGGCACCATAACAAAGAG \\
\hline \multirow[t]{2}{*}{ PcMPK7 } & Fwd: GCCTCTTACTTGTCTCCACG \\
\hline & Rev: TGTTGAGGAAACTGGAAAGC \\
\hline \multirow[t]{2}{*}{ РсMРК9-1 } & Fwd: TCCTTCGGCTGCTACATCAC \\
\hline & Rev: AAATGAAGCCATGCCCTTGT \\
\hline \multirow[t]{2}{*}{ PсMPK9-2 } & Fwd: ACAGGAGCGACCATTGAACC \\
\hline & Rev: GGACTGTTACAGAGCACCCT \\
\hline \multirow[t]{2}{*}{ PcMPK16 } & Fwd: ACCAAACGCTCTTCAGGTAG \\
\hline & Rev: CTATTCGCTAAACTTCCTCC \\
\hline \multirow[t]{2}{*}{ PcMPK20 } & Fwd: TGTGGGCTTGTGATAAGGAG \\
\hline & Rev: ACCCGACTCTTTGAATGCTC \\
\hline \multirow[t]{3}{*}{ PcMPK18 } & Fwd: CTTGTCATTCGGAGTGGGTCT \\
\hline & Rev: TACAATCACTCCACCCTCCC \\
\hline & Primers for qRT-PCR analysis of $P c M P K s$ \\
\hline \multirow[t]{2}{*}{ PcMPK4-1 } & Fwd: GGCTTATCACAGAGTTAA \\
\hline & Rev: AACACTAATGCGTCTATG \\
\hline \multirow[t]{2}{*}{ PcMPK4-2 } & Fwd: GTTGCCATTAAGAAGATTG \\
\hline & Rev: TGTAGACATCATTGAAGTT \\
\hline \multirow[t]{2}{*}{ PcMPK13 } & Fwd: CGTATAACTGTTGAGGAA \\
\hline & Rev: GTTGAAGTGTAGAGACTC \\
\hline \multirow[t]{2}{*}{ PcMPK3 } & Fwd: GAGTATGCTTGATTTGAAA \\
\hline & Rev: CCACCTAAGTAATGATGTA \\
\hline \multirow[t]{2}{*}{ РсMPK6 } & Fwd: ATATTCAGCAACTTCCTC \\
\hline & Rev: ATCTTCAACTGTAATTCTCT \\
\hline \multirow[t]{2}{*}{ PcMPK1 } & Fwd: AAGTTGCGATTAAGAAGA \\
\hline & Rev: CTCATACACCAAGTAGAC \\
\hline \multirow[t]{2}{*}{ PcMPK7 } & Fwd: GGATGTTATGATGCCTAT \\
\hline & Rev: TGAGTGAAGATACTTGAG \\
\hline \multirow[t]{2}{*}{ РсMРК9-1 } & Fwd: TTGAGTTGATGGAATCTG \\
\hline & Rev: AGTCTGAATTAGCAAGTATA \\
\hline \multirow[t]{2}{*}{ PcMPK9-2 } & Fwd: AAACAATGATGTGGAAAG \\
\hline & Rev: TCTTACACCTATACACTTC \\
\hline PcMPK16 & Fwd: AACCAAAGAAGATGTAAGA \\
\hline & Rev: ATAGTGCTCCTCAAGATA \\
\hline PcMPK20 & Fwd: CCAGTTATTCCACTTGAA \\
\hline & Rev: ATTCTTGTATGCCTCTTC \\
\hline PcMPK18 & Fwd: TCTAAGACATCCTGATATTG \\
\hline & Rev: CCTTGATAACTTGGTGAA \\
\hline$\beta$-actin & Fwd: CCTGGACTATGAGCAAGAAC \\
\hline & Rev: AATGAGTGATGGTTGGAAGAG \\
\hline
\end{tabular}

